
$\$$ Research Square
Preprints are preliminary reports that have not undergone peer review.
They should not be considered conclusive, used to inform clinical practice, or referenced by the media as validated information.

\title{
CircRNA Expression Patterns and circRNA-miRNA-mRNA Networks During CV-A16 Infection of SH-SY5Y Cells
}

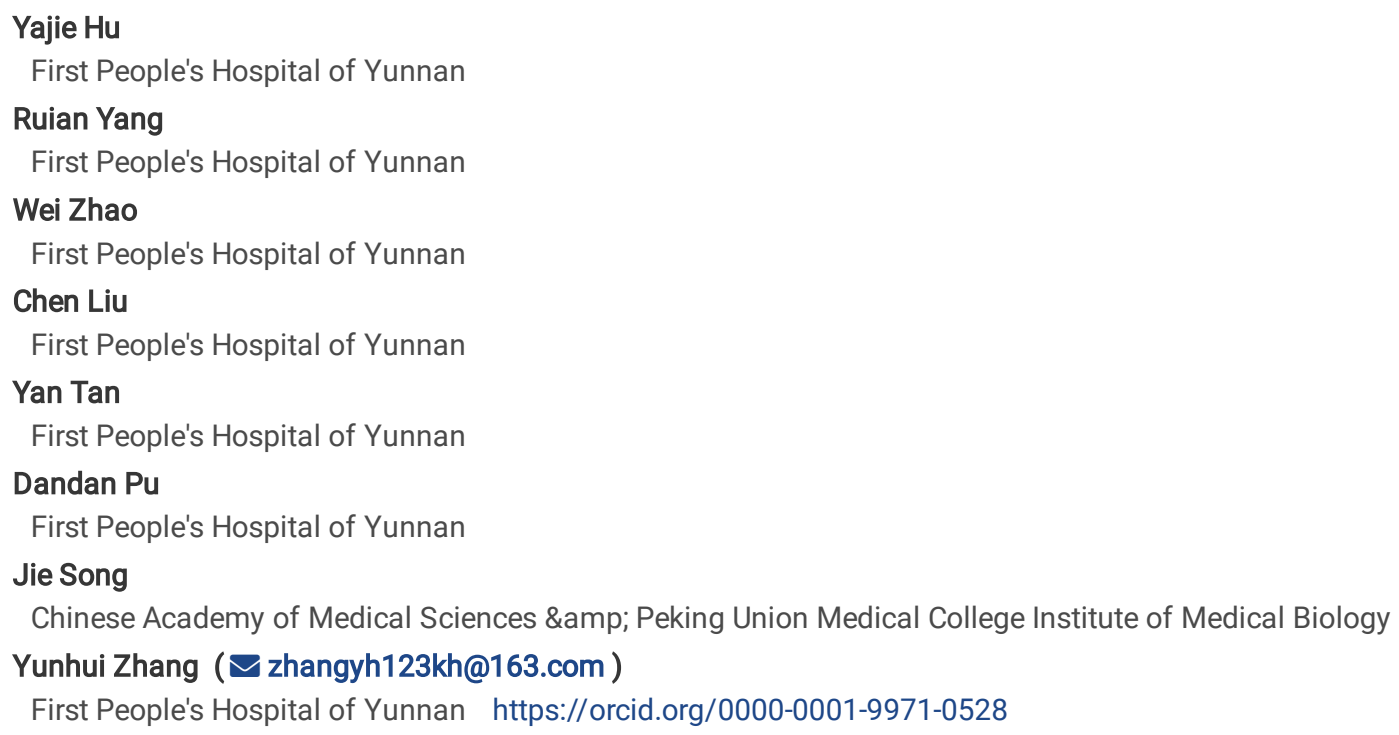

Keywords: Coxsackievirus A16 (CV-A16), RNA sequencing (RNA-seq), circular RNAs (circRNAs), competing endogenous RNA (ceRNA) network Posted Date: March 10th, 2021

DOI: https://doi.org/10.21203/rs.3.rs-253548/v1

License: (c) (1) This work is licensed under a Creative Commons Attribution 4.0 International License. Read Full License

Version of Record: A version of this preprint was published at Archives of Virology on August 19th, 2021. See the published version at https://doi.org/10.1007/s00705-021-05190-z. 


\section{Abstract}

Coxsackievirus A16 (CV-A16) has caused worldwide epidemics of hand, foot, and mouth disease (HFMD), particularly among infants and preschool children. Circular RNAs (circRNAs) are a recently identified class of noncoding RNA molecules that participate in the occurrence and development of numerous infectious diseases. To date, the function of circRNAs has been a heavily researched topic, and their role in CV-A16 infection has not been fully elucidated. In this study, the viral effects of CV-A16 on the cellular circRNA transcriptome were investigated using next-generation sequencing technology. In this study, a total of 8726,8611 and 6826 circRNAs were identified in the control, CV-A16-12 h and CV-A16-24 h groups, respectively. Further analysis showed that 1769 and 1192 circRNAs were differentially expressed in the CV-A16-12 h and CV-A16-24 h groups, respectively. Moreover, the common differentially expressed circRNAs were screened out and used for functional annotation analysis, which demonstrated that the parent genes of differentially expressed circRNAs might be associated with the viral infection process, especially the "Immune system process" in $\mathrm{GO}$ analysis and the "Inflammation mediated by chemokine and cytokine signaling pathway" in KEGG analysis. Additionally, 6 dysregulated circRNAs were selected for validation and were verified to be consistent with the sequencing results. Additionally, we further constructed a circRNAmiRNA-mRNA regulatory network that suggested the putative function of circRNAs as cellular miRNA decoys to indirectly regulate gene expression during CV-A16 infection, especially the hsa_circ_0004447/hsa-miR-942-5p/MMP2, hsa_circ_0078617/hsa-miR-6780b-5p/MMP2 and hsa_circ_0078617/hsa-miR-5196-5p/MMP2 regulatory axes. Considering all of these results, to the best of our knowledge, this study is the first to present a comprehensive overview of circRNAs induced by CV-A16, and this research demonstrated that a network of enriched circRNAs and circRNA-associated competitive endogenous RNAs (ceRNAs) is involved in the regulation of CV-A16 infection, thereby helping to elucidate the mechanisms underlying CV-A16-host interactions.

\section{Introduction}

Hand, foot, and mouth disease (HFMD) is a common infectious illness in infants and young children under 5 years of age that includes such symptoms as $3 \sim 4$ days of fever, a reduced appetite and the appearance of maculopapular or vesicular rash on the mouth, hands, feet and/or buttocks $[7,27]$. The two principal pathogenic agents of HFMD are enterovirus 71 (EV-A71) and coxsackievirus A16 (CV-A16)[2, 11]. In general, EVA71 frequently contributes to most severe cases and fatalities accompanied by serious complications especially those involving the central nervous system (CNS)[4], while CV-A16 infection usually results in a mild outcome[11, 22]. Hence, most studies have focused on EV-A71, and prophylactic EV-A71 vaccines developed by three companies (Beijing Vigoo Biological, Sinovac Biotech Co. Ltd, ant the Institute of Medical Biology) have all shown high efficacy and satisfactory safety in providing protection against EV-A71-associated diseases[2]. Unfortunately, these vaccines do not have cross-strain protective activity against HFMD induced by other enteroviruses, including CV-A16[2, 4]. However, in the past several decades, the number of clinical cases caused by CV-A16 was observed to increase steadily in the Asia-Pacific region[24]. Moreover, CV-A16 infections have also been reported to have more severe CNS complications, such as aseptic meningitis, cerebellar ataxia, poliomyelitis-like paralysis, acute brainstem encephalitis, cardiopulmonary failure, and fulminant neurogenic pulmonary edema[24,35]. Importantly, no antiviral therapies or vaccines for CVA16 are currently available yet; therefore, several vaccine companies and academic institutions are launching projects to develop monovalent or bivalent CV-A16 vaccines.

Circular RNAs (circRNAs) are a novel class of noncoding RNAs characterized by the formation of covalently closed continuous loops without $5^{\prime}$ to $3^{\prime}$ polarities and poly(A) tails[21,28]. Numerous studies have clearly indicated that circRNAs are widely expressed in plants, animals and humans with tissue- and developmental-stage specific patterns $[5,19]$. More importantly, circRNAs are involved in the regulatory process of gene expression by serving such roles as microRNA (miRNA) sponges, regulators of splicing and transcription, platforms for the assembly of protein complexes, templates for translation, etc[6]. Therefore, circRNAs play an important role in many cellular processes, such as embryonic development, control of cell cycle, cellular senescence, cell signaling, and response to cellular stress[15]. The perturbation of circRNA expression might be correlated with various diseases, such as cancer, heart disease, neurological disorders, diabetes and atherosclerosis, and even viral diseases[13, 33]. Hence, in this study, deep circRNA sequencing technology was used to explore the potential functions of aberrantly expressed circRNAs in CV-A16-infected SHSY5Y cells and these results might help to elucidate the mechanisms governing the interactions between CV-A16 and the host, and meanwhile might provide new therapeutic targets against CV-A16 infection.

\section{Materials And Methods}

\subsection{Cell culture and virus inoculation}

The human neuroblastoma cell line SH-SY5Y, obtained from Jennino Biological Technology, was cultivated in Roswell Park Memorial Institute-1640 medium (RPMI-1640; Corning, USA) supplemented with $10 \%$ fetal bovine serum (FBS; Gibco, USA), streptomycin (100 mg/ml), and penicillin (100 $\mathrm{U} / \mathrm{ml}$ ) in a humidified atmosphere consisting of $5 \% \mathrm{CO}_{2}$ and $90 \%$ humidity at $37^{\circ} \mathrm{C}$. Cell passage was performed once cells reached $90 \%$ confluence by rinsing twice with phosphate buffered saline (PBS) and digesting with $1 \mathrm{ml}$ of $0.25 \%$ trypsin (Beyotime Biotechnology Co., China). Next, the single-cell suspension was placed into culture flasks or 6-well plates for further experiments. 
The virus CV-A16-G20 strain (subgenotype B, GenBank: JN590244.1), isolated from an HFMD patient in Guangxi, China in 2010, was applied in this study. Six-well tissue culture plates were seeded at $\sim 1 \times 10^{6} \mathrm{SH}-\mathrm{SY} 5 \mathrm{Y}$ cells/well. When the cells reached approximately $90 \%$ confluence, they were washed twice with PBS, and CV-A16 was incubated with the cells at a multiplicity of infection (MOI) of 1 . Following infection for $0 \mathrm{~h}, 12 \mathrm{~h}, 24 \mathrm{~h}$, the cells were collected and cells treated with CV-A16 for $0 \mathrm{~h}$ were defined as the control group.

\subsection{RNA extraction and circRNA sequencing}

Total RNA was extracted from three independent experiments of each group, which pooled into one group following the instructions of TRIzol Reagent (Invitrogen, USA). RNA integrity was analyzed with an Agilent 2100 Bioanalyzer (Agilent Technologies, USA), while RNA concentration was tested by a Qubit RNA Assay Kit in a Qubit Fluorometer (Invitrogen, USA). Furthermore, total RNA samples that met the following requirements were utilized for the subsequent experiments: total RNA level $>5 \mu \mathrm{g}$, RNA integrity number $(\mathrm{RIN}) \geq 7.0$ and a $28 \mathrm{~S}: 18 \mathrm{~S}$ ratio $\geq 1.5$. Finally, the Ribo-Zero ${ }^{\text {TM }}$ Magnetic Kit (Epicentre Technologies, USA) was used to remove ribosomal RNA (rRNA) and RNase R (Epicentre Technologies, USA) was used to remove linear RNA and enrich circular RNA.

Sequencing libraries were generated by the NEBNext Ultra RNA Library Prep Kit for Illumina (NEB, USA) according to the manufacturer's protocol. The libraries were controlled for quality and quantified using the BioAnalyzer 2100 system. Fragments were sequenced on the Illumina HiSeq2000 platform by using 150-bp paired-end reads. We removed adapters from the raw FASTQ files using Trim Galore. After 3' adapters were trimmed and low-quality reads were removed using Cutadapt software, the high-quality trimmed reads were used for analysis of circRNAs. We aligned the filtered sequence data to the human reference genome (GRCh37/hg19) obtained from the UCSC genome database (http://genome.ucsc.edu/) by TopHat2. The sequencing data that could not be aligned to the reference genome directly were subjected to subsequent circRNA analysis by recognition of the reverse splicing event using Find_circ (v1.0) and CIRCexplorer2 software. We normalized circRNA contents as the number of uniquely mapped fragments per kilobase of exon per million fragments mapped (FPKM).

\subsection{Bioinformatic analyses}

\subsubsection{Detection and characterization of circRNAs overall data}

Raw junction reads for all samples were normalized by total read number and were log2 transformed. Next, the circRNAs distribution on human chromosomes could be explored, and the positive and negative strands of circRNAs located on different chromosomes were also analyzed. The log2-ratio was used for quantile normalization. circRNA sequencing data were deposited in the NCBI Gene Expression Omnibus (GEO) under accession number GSE151890.

\subsubsection{Identification of differently expressed circRNAs}

The Limma (v3.32.10) package of R software was used to analyze the differential expression of circRNAs. circRNAs with fold changes $>2$ and $P$ values $<0.05$ were selected as significantly differential expressions. According to the source of circRNA formation, the classification of circRNAs was mainly divided into 4 categories, namely, exonic, intronic, antisense and intragenic, which are summarized in a pie chart.

\subsubsection{Screening of the common differentially expressed circRNAs during CV-A16 infection}

To further identify the majority of CV-A16 dependent circRNAs that were induced in both the CV-A16-12 h and CV-A16-24 h groups. Venn diagram analysis was performed. Furthermore, hierarchical clustering was performed to obtain an overview of the expression profiles of circRNAs among the samples using these selected common circRNAs.

\subsubsection{Functional analysis of the differential circRNAs and their host genes}

To investigate the potential functions of the differentially expressed circRNA parent genes, Gene Ontology (GO) and Kyoto Encyclopedia of Genes and Genomes (KEGG) Pathway based on the Database for Annotation, Visualization and Integrated Discovery (DAVID) were used in this study. GO terms provided information about the biological process (BP), cellular component (CC) and molecular function (MF), whereas KEGG analysis was performed to explore the pathways related to circRNA-hosting genes.

\subsubsection{Construction of the circRNA-miRNA-mRNA coexpression network}

To establish a circRNA-miRNA-mRNA network, we first searched for miRNA response elements to circRNAs using miRanda and RNAhybrid software. A hit between circRNAs and a target miRNA with a perfect seed match was considered for a miRanda score of 150 or higher, and meanwhile the RNAhybrid energy could be less than - 25. Then, the putative miRNAs were further applied to the prediction of their target genes based on TargetScan and miRDB algorithms. In order to clarify the meaning of the target genes corresponding to miRNAs, we screened the miRNA target genes according to the meaning regulated by circRNAs, but the screening of the miRNA target gene would satisfy at least a perfect seed-match sequences between miRNAs and target genes in TargetScan, and a target score $\geq 80$ in miRDB.

2.4. Differentially expressed circRNAs validation by quantitative real-time polymerase chain reaction analysis (qRT-PCR) method

Page $3 / 13$ 
We conducted qRT-PCR to validate the results from our RNA sequencing approach, focusing on these circRNAs which significantly different in the expression levels. Total RNA from cells and tissue samples was isolated using TRIzol Reagent (Invitrogen, USA). Specific divergent primers spanning the back-splice junction site of circRNAs were designed by Sangon Biotech (Shanghai, China) and listed in Table 1. GAPDH, a housekeeping gene, was employed as an internal control. To quantify the amount of circRNA, complementary DNA (cDNA) was synthesized using

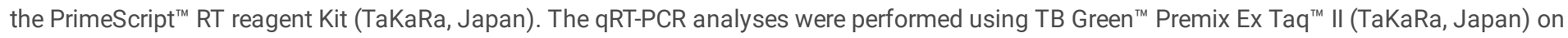
an ABI 7500 real-time PCR system (Applied Biosystems, USA). The formula $2^{-\triangle \Delta C t}$ was used to analyze the relative expression levels of different circRNAs.

Table 1

Primers used for qRT-PCR analysis of circRNA and reference genes.

\begin{tabular}{|c|c|}
\hline CircBase ID & Primer sequences \\
\hline \multirow[t]{2}{*}{ hsa_circ_0000205 } & Forward primer: 5'-AGTTGGCTCTCACTGCTTCT-3' \\
\hline & Reverse primer: 5'-GGTCACTCCTGCAATAAGACT-3' \\
\hline \multirow[t]{2}{*}{ hsa_circ_0002485 } & Forward primer: 5'-CGTTTTCAGCGTGACAAGGA-3' \\
\hline & Reverse primer: 5'-CCAGCTGGTACCTCTTACTGT-3' \\
\hline \multirow[t]{2}{*}{ hsa_circ_0007059 } & Forward primer: 5'-GAGACAGTAGCCATCCAGC-3' \\
\hline & Reverse primer: 5'-TGATCTGAGTCCAGGTGTT-3' \\
\hline \multirow[t]{2}{*}{ hsa_circ_0002483 } & Forward primer: 5'-TGCCAAAAGGATTTCTAAACCAGT-3' \\
\hline & Reverse primer: 5'-TTGGGGTCAAGGTAAGCAGC-3' \\
\hline \multirow[t]{2}{*}{ hsa_circ_0002301 } & Forward primer: 5'-TATATGGTCAACTGCAACTTGGC-3' \\
\hline & Reverse primer: 5'-TCACATGTCTCCACCCTTGT-3' \\
\hline \multirow[t]{2}{*}{ hsa_circ_0002141 } & Forward primer: 5'-TCATTGTCAAGAGAGCAGATACT-3' \\
\hline & Reverse primer: 5'-TCCTTGTCTTTTCTGCATCTTGA-3' \\
\hline \multirow[t]{2}{*}{ GAPDH } & Forward primer: 5'-ATGAGGTCCACCACCCTGTT-3' \\
\hline & Reverse primer: 5'-CTCAAGGGCATCCTGGGCTA-3' \\
\hline
\end{tabular}

\subsection{Statistical analysis}

All of the qRT-PCR experiments were conducted in triplicate and all the results were reported as the mean \pm SD. Differences between two groups were analyzed with Student's t-test by using SPSS 22.0 (IBM-SPSS, USA), and a $P$-value $<0.05$ indicated a statistically significant difference.

\section{Results}

\subsection{Overview of circRNA expressions}

In this study, we identified circRNAs in CV-A16-infected SH-SY5Y cells and uninfected SH-SY5Y cells using RNA-Seq data generated from rRNAdepleted samples. There were 8726, 8611 and 6826 circRNAs obtained in the control, CV-A16-12 h and CV-A16-24 h groups, respectively. Moreover, the genomic loci from which circRNAs are derived were observed to be widely distributed across the chromosomes, except the $Y$ chromosome, and the positive and negative strands of circRNAs located on different chromosomes were also marked in Fig. 1. Thus, these differences among different groups indicated that CV-A16 infection triggered changes in circRNA in SH-SY5Y cells.

\subsection{Analysis of differentially expressed circRNAs}

Differential expression analysis showed that compared with the controls, 1769 differentially expressed circRNAs were found in the CV-A16-12 $\mathrm{h}$ group, of which 821 were upregulated and 948 were downregulated, while 1192 differentially expressed circRNAs were found in the CV-A16-24 $\mathrm{h}$ group, of which 653 were upregulated and 539 were downregulated (Fig. 2A). Subsequently, according to the source of the circRNA formation, the most significant differentially expressed circRNAs were transcribed from the exons of coding proteins (Fig. 2B). To further investigate the key roles of these distinct circRNAs in the progression of CV-A16 infection, the overlapping differentially expressed circRNAs in the CV-A16-12 h group and CV-A16-24 h group were screened out. A Venn diagram revealed that the cross-section represented 554 common circRNAs in each sample, and the independent section represents the number of sample-specific circRNAs during CV-A16 infection (Fig. 2C). Furthermore, these common circRNAs were utilized to perform hierarchical clustering analysis, which illustrated the distinguishable circRNA expression pattern among the samples (Fig. 2D). Therefore, the above data suggested that the changes in numerous circRNAs could be inducted in the process of CV-A16 infection, and 
the common differentially expressed circRNAs at different time points of CV-A16 infection might be the key regulatory factors in the pathogenesis of CV-A16.

\subsection{Biomathematically predicted biological function of host linear transcripts}

To investigate the potential functional implications of the circRNAs, we performed functional enrichment analysis for the parent genes of these common differentially expressed circRNAs. The GO terms and KEGG pathway analysis were presented and ranked by $P$-value. The results showed that for GO-BP terms, cellular process, metabolic process, biological regulation, localization, response to stimulus, signaling, developmental process, multicellular organismal process, biological adhesion, locomotion, immune system process, reproductive process, interspecies interaction between organisms, reproduction, biological phase, multi-organism process, and biomineralization were enriched (Fig. 3A). For GO-MF terms, the majority of the enriched terms were related to binding, catalytic activity, molecular function regulator, transporter activity, molecular transducer activity, molecular adaptor activity, structural molecule activity, and translation regulator activity (Fig. 3B). For GO-CC enrichment terms, the host linear transcripts of differentially expressed circRNAs were closely involved in cellular anatomical entity, intracellular, and protein-containing complex were enriched (Fig. 3C). Additionally, KEGG pathway analysis demonstrated that the top 10 regulated pathways primarily contained Gonadotropinreleasing hormone receptor pathway, EGF receptor signaling pathway, Wnt signaling pathway, FGF signaling pathway, Angiogenesis, Integrin signaling pathway, Inflammation mediated by chemokine and cytokine signaling pathway, PDGF signaling pathway, CCKR signaling map, and p53 pathway (Fig. 4). Hence, the results implied that the vital biological function regulated by the host linear transcripts of differentially expressed circRNAs might be closely associated with the pathogenic mechanism of CV-A16 infection.

\subsection{Prediction of circRNA-miRNA-target gene associations}

A large number of reports have shown that circular RNAs affect miRNA-mediated regulation of gene expression through miRNA sequestration[20]. In order to better excavate the circRNAs involved in the pathogenic mechanism of CV-A16 infection, we selected circRNAs related to "Immune system process" in GO-BPs analysis and circRNAs related to "Inflammation mediated by chemokine and cytokine signaling pathway" in KEGG analysis. Detailed information on these circRNAs was listed in Table 2 and Table 3, respectively. Then, the potential circRNA-binding miRNAs were predicted based on the miRanda and RNAhybrid algorithm. Moreover, to further elucidate the circRNA function, the target genes of the above putative miRNAs were predicted using TargetScan and miRDB. The final result of the prediction was used to draw a circRNA-miRNA-mRNA regulatory network map. It was seen that 5 circRNAs, 69 miRNAs and 17 mRNAs were in the "Immune system process"-related network (Fig. 5A), while 9 circRNAs, 134 miRNAs and 23 mRNAs were in the "Inflammation mediated by chemokine and cytokine signaling pathway"-related network (Fig. 5B). Taken together, the regulatory relationships among circRNAs, miRNAs and mRNAs might prompt that dysregulated circRNAs exerted a critical function during the occurrence and development of CV-A16 infection. In addition, innate antiviral and inflammatory responses can immediately be elicited upon viral infection[1, 14]. These responses must be finely regulated to prevent viral dissemination and present appropriate effective immune responses[18]. Therefore, we further searched for the targeted genes that co-existed in the two network diagrams, and the results showed that there were 16 genes (Fig. 5C). Among these 16 genes, MMP2 has attracted our attention. Subsequently, the network axis of MMP2 regulated by circRNA was screened, including hsa_circ_0004447/hsa-miR-942-5p/MMP2, hsa_circ_0078617/hsa-miR-6780b-5p/MMP2 and hsa_circ_0078617/hsa-miR-5196-5p/MMP2 (Fig. 5C), which simultaneously participated in "Immune system process" and "Inflammation mediated by chemokine and cytokine signaling pathway" during CV-A16 infection. Thus, the 3 key circRNA-associated ceRNA networks pointed out a new direction for our future research.

Table 2

Differentially expressed circRNAs associated with "Immune system process" in GO-BPs analysis.

\begin{tabular}{|c|c|c|c|c|c|c|c|c|}
\hline & CV-A16-12 h & & CV-A16-24 h & & CHROM & GeneName & CircType & SeqLength \\
\hline AccID & Log2FC & Style & Log2FC & Style & & & & \\
\hline $\begin{array}{l}\text { chr13_46728984_46723221_+5763- } \\
\text { LRCH1 }\end{array}$ & 20 & Up & 1 & Up & chr13 & LRCH1 & Exonic & 248 \\
\hline $\begin{array}{l}\text { chr4_73125312_73118688_-6624- } \\
\text { ANKRD17 }\end{array}$ & 2 & Up & -2.584962501 & Down & chr4 & ANKRD17 & Exonic & 954 \\
\hline $\begin{array}{l}\text { chr2_195681050_195680032_+1018- } \\
\text { SLC39A10 }\end{array}$ & 20 & Up & -20 & Down & chr2 & SLC39A10 & Exonic & 1019 \\
\hline $\begin{array}{l}\text { chr6_47286595_47283938_-2657- } \\
\text { TNFRSF21 }\end{array}$ & 1.378511623 & Up & -20 & Down & chr6 & TNFRSF21 & Exonic & 1147 \\
\hline $\begin{array}{l}\text { chr5_108798389_108768093_+30296- } \\
\text { FER }\end{array}$ & 20 & Up & -20 & Down & chr5 & FER & Exonic & 412 \\
\hline
\end{tabular}


Table 3

Differentially expressed circRNAs associated with "Inflammation mediated by chemokine and cytokine signaling pathway" in KEGG analysis.

\begin{tabular}{|c|c|c|c|c|c|c|c|c|}
\hline & CV-A16-12 h & & CV-A16-24 h & & CHROM & GeneName & CircType & SeqLength \\
\hline AcclD & Log2FC & Style & Log2FC & Style & & & & \\
\hline $\begin{array}{l}\text { chr10_94032252_94030683_+1569- } \\
\text { PLCE1 }\end{array}$ & 20 & Up & -20 & Down & chr10 & PLCE1 & Exonic & 1570 \\
\hline $\begin{array}{l}\text { chr16_69677335_69626403_+50932- } \\
\text { NFAT5 }\end{array}$ & 20 & Up & -20 & Down & chr16 & NFAT5 & Exonic & 1563 \\
\hline $\begin{array}{l}\text { chr16_68174514_68121987_+52527- } \\
\text { NFATC3 }\end{array}$ & 1.736965594 & Up & -3.321928095 & Down & chr16 & NFATC3 & Exonic & 1812 \\
\hline $\begin{array}{l}\text { chr21_33432871_33414888_+17983- } \\
\text { IFNGR2 }\end{array}$ & 20 & Up & -20 & Down & chr21 & IFNGR2 & Exonic & 806 \\
\hline $\begin{array}{l}\text { chr1_243843282_243637611_-205671- } \\
\text { AKT3 }\end{array}$ & -20 & Down & 20 & Up & chr1 & AKT3 & Exonic & 673 \\
\hline $\begin{array}{l}\text { chr21_33349543_33345246_+4297- } \\
\text { IFNAR1 }\end{array}$ & 20 & Up & -20 & Down & chr21 & IFNAR1 & Exonic & 470 \\
\hline $\begin{array}{l}\text { chr2_46010517_46001404_+9113- } \\
\text { PRKCE }\end{array}$ & 20 & Up & -20 & Down & chr2 & PRKCE & Exonic & 614 \\
\hline $\begin{array}{l}\text { chr2_39058804_39051144_-7660- } \\
\text { SOS1 }\end{array}$ & 20 & Up & -20 & Down & chr2 & sos1 & Exonic & 651 \\
\hline $\begin{array}{l}\text { chr6_161049979_161034259_+15720- } \\
\text { MAP3K4 }\end{array}$ & 1.94753258 & Up & -3.169925001 & Down & chr6 & MAP3K4 & Exonic & 1555 \\
\hline
\end{tabular}

\subsection{Validation of the differential expression levels of circRNAs}

In order to verify the reliability of the circRNA-seq results, 6 circRNAs showed differential expression, consisting of hsa_circ_0000205, hsa_circ_0002485, hsa_circ_0007059, hsa_circ_0002483, hsa_circ_0002301 and hsa_circ_0002141, were selected for validation by qRT-PCR. And the results verified that the data obtained by qRT-PCR was highly consistent with the high-throughput data for all of the 6 circRNAs (Fig. 6 ).

\section{Discussion}

circRNAs were originally thought to be by-products of aberrant splicing with little functional potential[10]. Novel bioinformatic approaches coupled with biochemical enrichment strategies and deep sequencing have enabled comprehensive studies of circRNAs to be performed[9], and several studies have addressed the identification, characterization, and function of circRNAs during viral infection[32]. For example, human cytomegalovirus (HCMV) could cause significant changes in host circRNAs and it was found that circSP100 potentially bound to 10 HCMVencoding proteins, which further influenced the process of HCMV infection[8]. The host circRNA-miRNA-mRNA network was significantly changed in human lung adenocarcinoma epithelial (Calu-3) cells infected with the highly pathogenic Middle East respiratory syndrome coronavirus (MERS$\mathrm{CoV}$ ), and circFNDC3B and circCNOT were also verified to reduce the MERS-CoV load by modulating various biological pathways, including the mitogen-activated protein kinase (MAPK) and ubiquitination pathways[36]. Nevertheless, the properties and potential roles of circRNAs during CVA16 infection have not been thoroughly elucidated to date. In this study, we applied a next-generation sequencing technique to systematically analyze circRNA expression profiles in SH-SY5Y cells infected with CV-A16. In this way, thousands of circRNAs were identified from diverse genomic locations at different infectious times. Moreover, among these circRNAs, it was found that there were 1769 and 1192 showed differentially expressions in the CV-A16-12 h and CV-A16-24 h groups, respectively. While a small fraction of these circRNAs were intronic, nearly more than $90 \%$ of them originated from exonic. The large number of different circRNAs presented at different times indicated that the transcription of circRNAs was very active after CV-A16 infection. Functional circRNAs can directly affect host gene expression by regulating transcription or interfering with splicing, mainly due to that circRNAs are usually generated from host genes through backsplicing. Thus, the function of these dysregulated circRNAs was assessed by GO and KEGG enrichment analysis with their host genes. Furthermore, to further investigate the key circRNAs involved in the process of CV-A16 infection, we screened out only the common differentially expressed circRNAs during CV-A16 infection at different time points. The host genes that were related to these 554 overlapping dysregulated circRNAs participated in various biological processes, such as cellular process, metabolic process, biological regulation, localization, response to stimulus, signaling, developmental process, multicellular organismal process, biological adhesion, locomotion, immune system process, reproductive process, interspecies interaction between organisms, reproduction, biological phase, multi-organism process, and biomineralization, most of which were associated with initiation and progression of viral infection. Additionally, the KEGG annotation was also showed that these circRNAs might primarily regulate Gonadotropin-releasing hormone receptor pathway, EGF receptor signaling pathway, Wnt signaling pathway, FGF signaling pathway, Angiogenesis, Integrin signaling pathway, Inflammation mediated by chemokine and cytokine signaling pathway, PDGF signaling pathway, CCKR signaling map, and p53 pathway, which 
were involved in host regulatory mechanisms during virus infection. For instance, EGF receptor inhibitors have been found to inhibit hepatitis B virus (HBV) and hepatitis $\mathrm{C}$ virus (HCV) replication via downregulation of signal transducers and activators of transcription 3 (STAT3) phosphorylation[12]. Activation of the Wnt/ $\beta$-catenin pathway increased influenza virus mRNA and virus production in in vitro in mouse lung epithelial E10 cells and mRNA expression of influenza virus genes in vivo in mouse lungs[25]. Thence, the above results prompted that the alterations of circRNAs induced by CV-A16 infection in SH-SY5Y cells might play important regulatory roles in CV-A16-infected SH-SY5Y cells by directly influencing host biological functions and pathways. Next, qRT-PCR methods were utilized to test and verify the reliability of RNA-seq data, which showed that the expression of selected random circRNAs was consistent with the sequencing results, although the fold-change was slightly different from the sequencing result.

As highly conserved endogenous RNAs, many circRNAs harbor abundant miRNA binding sites, indicating that they can sponge corresponding miRNAs and thus function as competing endogenous RNAs (ceRNAs) to regulate gene expression[30]. For instance, circRNA Cdr1as functioned as a ceRNA to promote hepatocellular carcinoma progression via sponging miR-1270 and enhancing the expression of AFP[29]. The circRNA_15698/miR-185/TGF-beta1 axis aggravated the extracellular matrix of diabetic nephropathy mesangial cells, which promoted diabetic nephropathy pathogenesis[16]. Thus, in order to investigate the potential functions of key circRNAs, we focused on the major significantly changed GO terms (i.e., "Immune system process") and most correlated pathways (i.e., "Inflammation mediated by chemokine and cytokine signaling pathway"), and a circRNA-associated-ceRNA network was constructed. Most viruses are controlled satisfactorily by the immune system with limited damage to host tissues[31]. However, during the evolution of the viruses, they also can obtain its survival in the host cell through an immune escape mechanism[26]. Thus, exploring the "Immune system process" induced by CV-A16 infection might provide more clues for understanding its pathogenic mechanism. In addition, accumulating evidence has reported that inflammation is a major driving force in virus infection[3, 17], and the development of neurological symptoms caused by CV-A16 is closely associated with inflammation of the nervous system. Hence, the investigation of "Inflammation mediated by chemokine and cytokine signaling pathway" could help us to further excavate potential mechanisms and therapeutic agents in CV-A16 infection progression. In the circRNA-miRNA-mRNA triple network map, it was seen that miRNA that potentially bind to the circRNA and the most likely target genes to each miRNA were identified. Among these complicated networks, we paid particularly close attention to the circRNAs that may act as ceRNAs to regulate the expression of MMP2, which has previously been reported to participate in the regulation of viral infections. For example, MMP2 could mediate viral clearance during HBV infection by cleaving membrane-bound CD100 into soluble CD100 from T cells[34]. Dengue virus could directly infect macrovascular endothelial cells and result in overproduction of MMP-2, which might contribute to the pathogenesis of severe dengue infection[23]. In the current study, it was discovered 3 circRNAs-regulated axes on MMP2, namely hsa_circ_0004447/hsa-miR-942-5p/MMP2, hsa_circ_0078617/hsa-miR-6780b-5p/MMP2 and hsa_circ_0078617/hsa-miR-5196-5p/MMP2. Thence, it gave us a clear direction to study the specific mechanism of these circRNAs in the pathogenesis of CV-A16 infection.

In conclusion, in this study, we identified a population of circRNAs differentially expressed in CV-A16-treated and CV-A16-untreated SH-SY5Y cells by high-throughput sequencing analysis and verified the expression of 6 dysregulated circRNAs by qRT-PCR. Further analysis revealed that these common differentially expressed circRNAs in different groups may exert a crucial role in viral pathogenicity by participating in the regulation of viral infection-associated biological processes and signaling pathways. Moreover, the establishment of a ceRNA network further outlined the regulatory function of circRNAs that could potentially restrict or facilitate EV-A71 infection through regulating gene expression, especially 3 circRNAs regulatory axes involved in MMP2 regulation. Thus, the results of this study may be helpful for future studies investigating the molecular functions of circRNAs in viral pathogenesis and virus-host interactions of CV-A16 infection.

\section{Declarations}

\section{Funding}

This work is financially supported by National Natural Sciences Foundations of China (32000128), Yunnan Applied Basic Research Projects (2019FB018 and 2018ZF006), Fundamental Research Funds for the Central Universities and PUMC Youth Fund (3332019004), Medical Reserve Talents of Yunnan Province Health and Family Planning (H-2017034 and H-2019061)هDoctoral Fund of the First People's Hospital of Yunnan Province (KHBS-2020-013) and Top young talents of Yunnan province ten thousand talents plan (Jie Song). The funders had no role in the study design, data collection and analysis, decision to publish, or preparation of the manuscript.

\section{Conflict of Interest:}

The authors declare that they have no conflicts of interest.

\section{Ethical approval:}

This article does not contain any studies with human participants or animals performed by any of the authors.

\section{References}


1. Aoshi T, Koyama S, Kobiyama K, Akira S, Ishii KJ (2011) Innate and adaptive immune responses to viral infection and vaccination. Curr Opin Virol 1:226-232

2. Aswathyraj S, Arunkumar G, Alidjinou EK, Hober D (2016) Hand, foot and mouth disease (HFMD): emerging epidemiology and the need for a vaccine strategy. Med Microbiol Immunol 205:397-407

3. Carty M, Guy C, Bowie AG (2021) Detection of Viral Infections by Innate Immunity. Biochem Pharmacol 183:114316

4. Chang YK, Chen KH, Chen KT (2018) Hand, foot and mouth disease and herpangina caused by enterovirus A71 infections: a review of enterovirus A71 molecular epidemiology, pathogenesis, and current vaccine development. Rev Inst Med Trop Sao Paulo 60:e70

5. Chen LL, Yang L (2015) Regulation of circRNA biogenesis. RNA Biol 12:381-388

6. Chen LL (2020) The expanding regulatory mechanisms and cellular functions of circular RNAs. Nat Rev Mol Cell Biol 21:475-490

7. Cox B, Levent F (2018) Hand, Foot, and Mouth Disease. JAMA 320:2492

8. Deng J, Huang Y, Wang Q, Li J, Ma Y, Qi Y, Liu Z, Li Y, Ruan Q (2020) Human Cytomegalovirus Influences Host circRNA Transcriptions during Productive Infection. Virol Sin

9. Dori M, Bicciato S (2019) Integration of Bioinformatic Predictions and Experimental Data to Identify circRNA-miRNA Associations. Genes (Basel) 10

10. Ebbesen KK, Hansen TB, Kjems J (2017) Insights into circular RNA biology. RNA Biol 14:1035-1045

11. Esposito S, Principi N (2018) Hand, foot and mouth disease: current knowledge on clinical manifestations, epidemiology, aetiology and prevention. Eur J Clin Microbiol Infect Dis 37:391-398

12. Gan CJ, Li WF, Li CN, Li LL, Zhou WY, Peng XM (2020) EGF receptor inhibitors comprehensively suppress hepatitis B virus by downregulation of STAT3 phosphorylation. Biochem Biophys Rep 22:100763

13. Gao JL, Chen G, He HQ, Wang J (2018) [CircRNA as a new field in human disease research]. Zhongguo Zhong Yao Za Zhi 43:457-462

14. Getts DR, Chastain EM, Terry RL, Miller SD (2013) Virus infection, antiviral immunity, and autoimmunity. Immunol Rev 255:197-209

15. Hsiao KY, Sun HS, Tsai SJ (2017) Circular RNA - New member of noncoding RNA with novel functions. Exp Biol Med (Maywood) 242:11361141

16. Hu W, Han Q, Zhao L, Wang L (2019) Circular RNA circRNA_15698 aggravates the extracellular matrix of diabetic nephropathy mesangial cells via miR-185/TGF-beta1. J Cell Physiol 234:1469-1476

17. Johnson RT (1971) Inflammatory response to viral infection. Res Publ Assoc Res Nerv Ment Dis 49:305-312

18. Koyama S, Ishii KJ, Coban C, Akira S (2008) Innate immune response to viral infection. Cytokine 43:336-341

19. Kristensen LS, Andersen MS, Stagsted LVW, Ebbesen KK, Hansen TB, Kjems J (2019) The biogenesis, biology and characterization of circular RNAs. Nat Rev Genet 20:675-691

20. Lee WJ, Moon J, Jeon D, Kim TJ, Yoo JS, Park DK, Lee ST, Jung KH, Park KI, Jung KY, Kim M, Lee SK, Chu K (2018) Possible epigenetic regulatory effect of dysregulated circular RNAs in epilepsy. PLoS One 13:e0209829

21. Li X, Yang L, Chen LL (2018) The Biogenesis, Functions, and Challenges of Circular RNAs. Mol Cell 71:428-442

22. Luo K, Rui J, Hu S, Hu Q, Yang D, Xiao S, Zhao Z, Wang Y, Liu X, Pan L, An R, Guo D, Su Y, Zhao B, Gao L, Chen T (2020) Interaction analysis on transmissibility of main pathogens of hand, foot, and mouth disease: A modeling study (a STROBE-compliant article). Medicine (Baltimore) 99:e19286

23. Luplertlop N, Misse D (2008) MMP cellular responses to dengue virus infection-induced vascular leakage. Jpn J Infect Dis 61:298-301

24. Mao Q, Wang Y, Yao X, Bian L, Wu X, Xu M, Liang Z (2014) Coxsackievirus A16: epidemiology, diagnosis, and vaccine. Hum Vaccin Immunother 10:360-367

25. More S, Yang X, Zhu Z, Bamunuarachchi G, Guo Y, Huang C, Bailey K, Metcalf JP, Liu L (2018) Regulation of influenza virus replication by Wnt/beta-catenin signaling. PLoS One 13:e0191010

26. Nelemans T, Kikkert M (2019) Viral Innate Immune Evasion and the Pathogenesis of Emerging RNA Virus Infections. Viruses 11

27. Saguil A, Kane SF, Lauters R, Mercado MG (2019) Hand-Foot-and-Mouth Disease: Rapid Evidence Review. Am Fam Physician 100:408-414

28. Salzman J (2016) Circular RNA Expression: Its Potential Regulation and Function. Trends Genet 32:309-316

29. Su Y, Lv X, Yin W, Zhou L, Hu Y, Zhou A, Qi F (2019) CircRNA Cdr1as functions as a competitive endogenous RNA to promote hepatocellular carcinoma progression. Aging (Albany NY) 11:8182-8203

30. Tay Y, Rinn J, Pandolfi PP (2014) The multilayered complexity of ceRNA crosstalk and competition. Nature 505:344-352

31. Thompson MR, Kaminski JJ, Kurt-Jones EA, Fitzgerald KA (2011) Pattern recognition receptors and the innate immune response to viral infection. Viruses 3:920-940

32. Xie H, Sun H, Mu R, Li S, Li Y, Yang C, Xu M, Duan X, Chen L (2021) The role of circular RNAs in viral infection and related diseases. Virus Res 291:198205

33. Yan L, Chen YG (2020) Circular RNAs in Immune Response and Viral Infection. Trends Biochem Sci 45:1022-1034

Page $8 / 13$ 
34. Yang S, Wang L, Pan W, Bayer W, Thoens C, Heim K, Dittmer U, Timm J, Wang Q, Yu Q, Luo J, Liu Y, Hofmann M, Thimme R, Zhang X, Chen H, Wang H, Feng X, Yang X, Lu Y, Lu M, Yang D, Liu J (2019) MMP2/MMP9-mediated CD100 shedding is crucial for inducing intrahepatic anti-HBV CD8 T cell responses and HBV clearance. J Hepatol 71:685-698

35. Yu W, Xu H, Yin C (2016) Molecular epidemiology of human coxsackievirus A16 strains. Biomed Rep 4:761-764

36. Zhang X, Chu H, Wen L, Shuai H, Yang D, Wang Y, Hou Y, Zhu Z, Yuan S, Yin F, Chan JF, Yuen KY (2020) Competing endogenous RNA network profiling reveals novel host dependency factors required for MERS-CoV propagation. Emerg Microbes Infect 9:733-746

\section{Figures}

\section{Control}

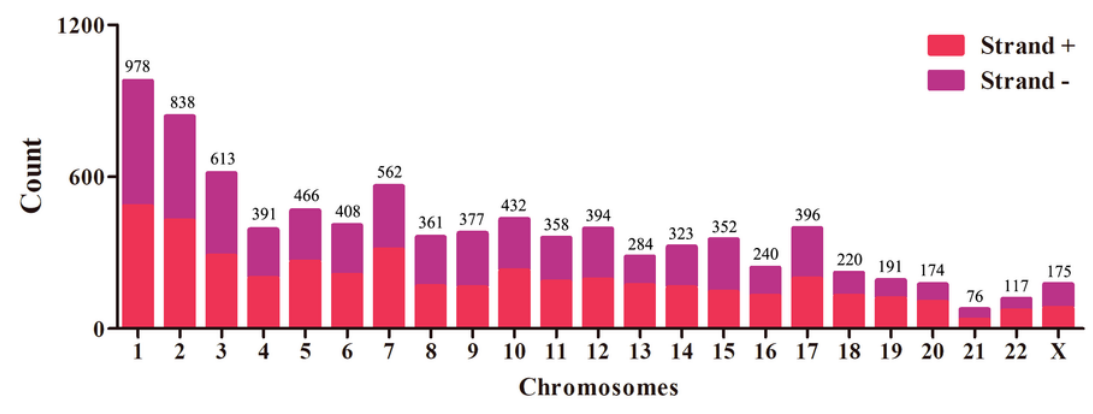

CV-A16-12h

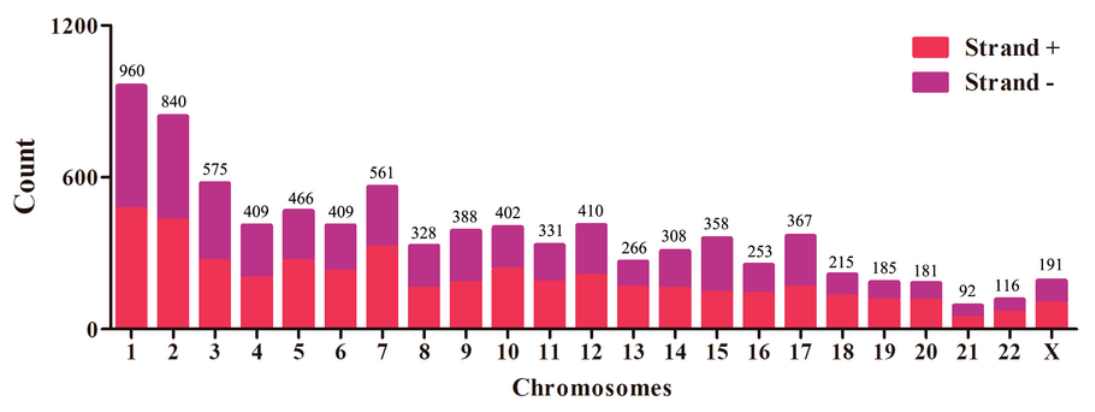

CV-A16-24h

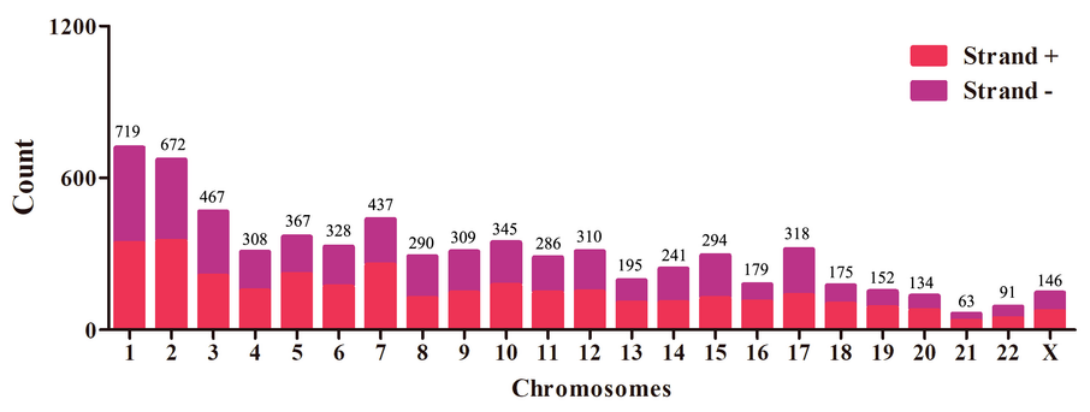

Figure 1

View of circRNAs expression profiles. Chromosomal distribution of the circRNAs in the control group and CV-A16-infected groups. 
A

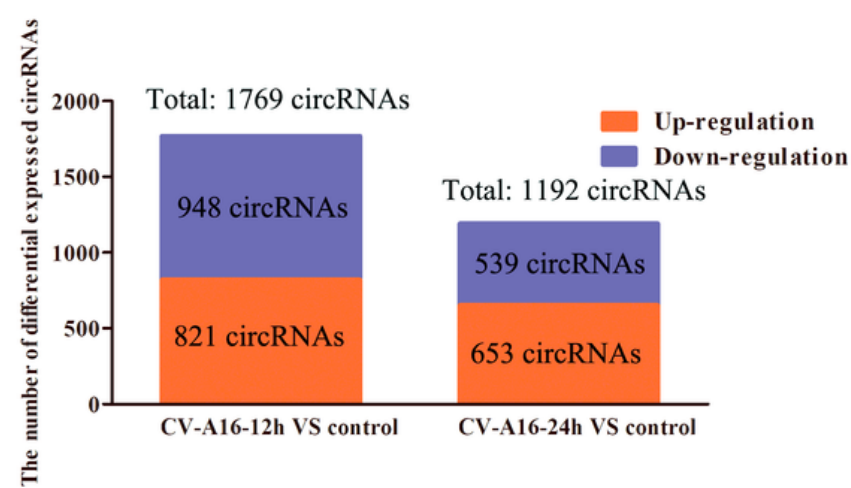

B

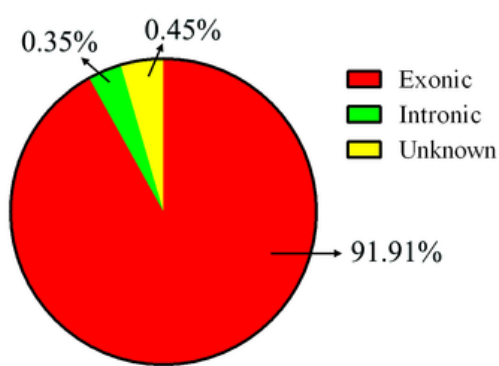

CV-A16-12h

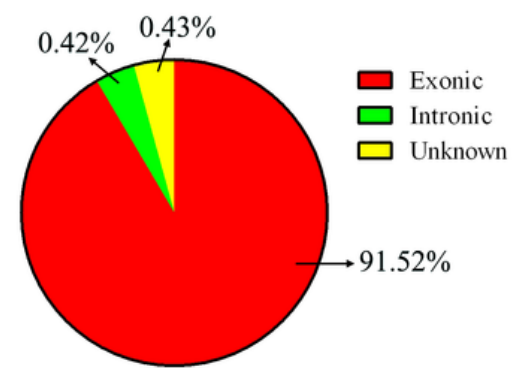

CV-A16-24h

$\mathrm{D}$

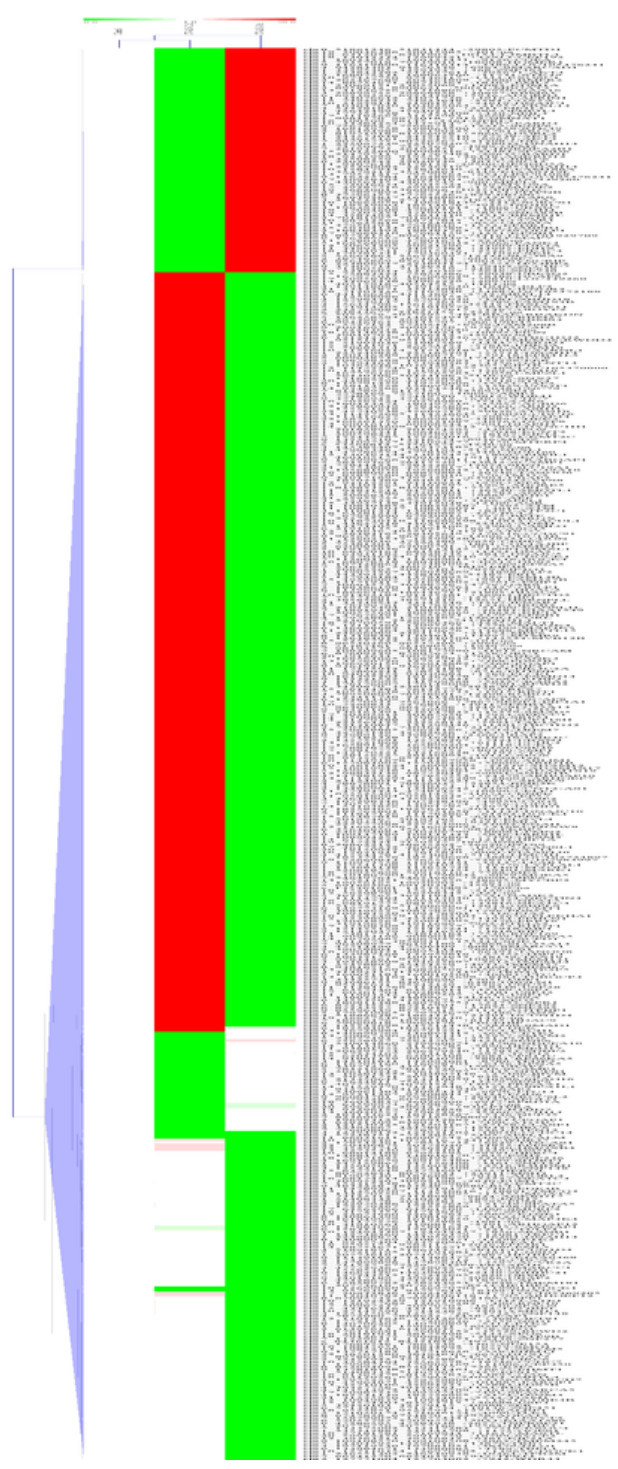

Figure 2

Differences and characterizations of circRNA expression profiles between the CV-A16-infected groups and the control group. (A) The counts of dysregulated differentially expressed circRNAs in CV-A16-infected groups. (B) The percentage of significantly differentially expressed circRNAs arising from different genomic loci was analyzed in a pie chart. (C) Venn diagram analysis of differentially expressed circRNAs in the CV-A16infected groups indicated similarities and differences. (D) Hierarchical clustering results of circRNA expression profiles among the CV-A16-infected groups and the control group. "Red" indicates high relative expression, and "green" indicates low relative expression. 


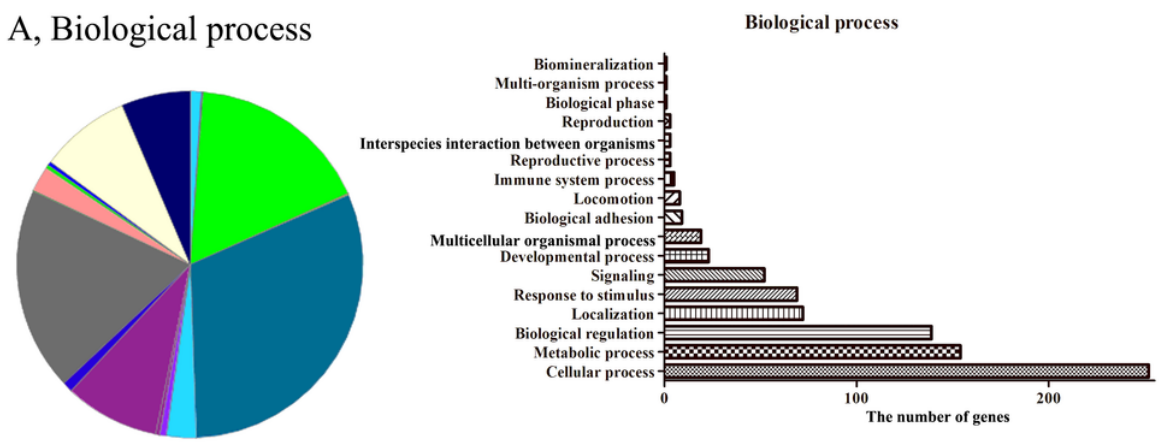

B, Molecular function
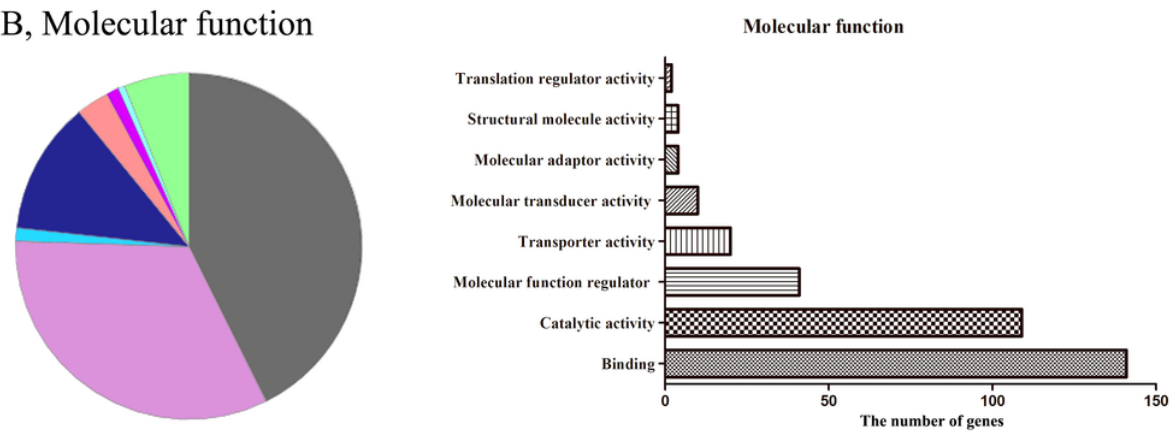

\section{C, Cellular components}
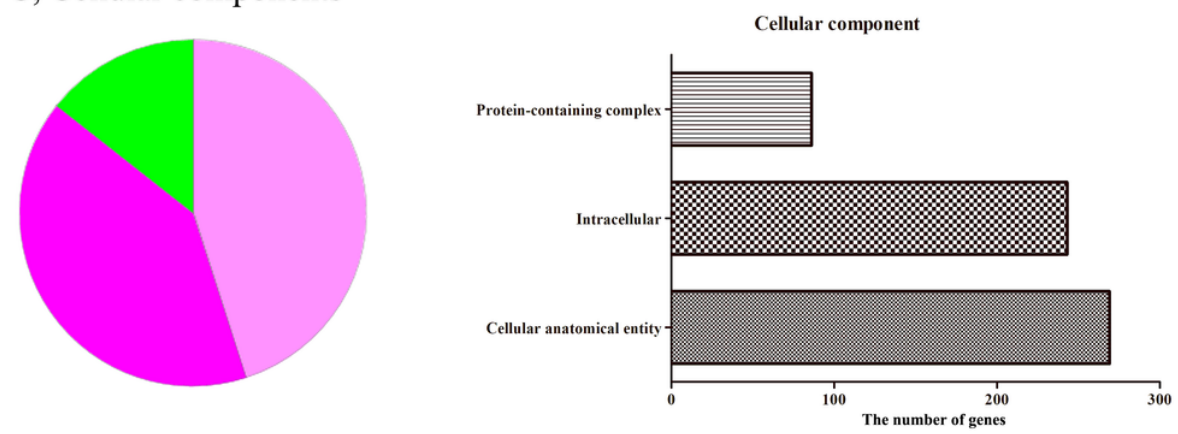

Figure 3

Enrichment map of GO annotation for the host genes of these common differentially expressed circRNAs. (A) Biological process. (B) Molecular function. (C) Cellular component. The meanings of the $X$ and $Y$ axes represented gene count and GO terms, respectively.
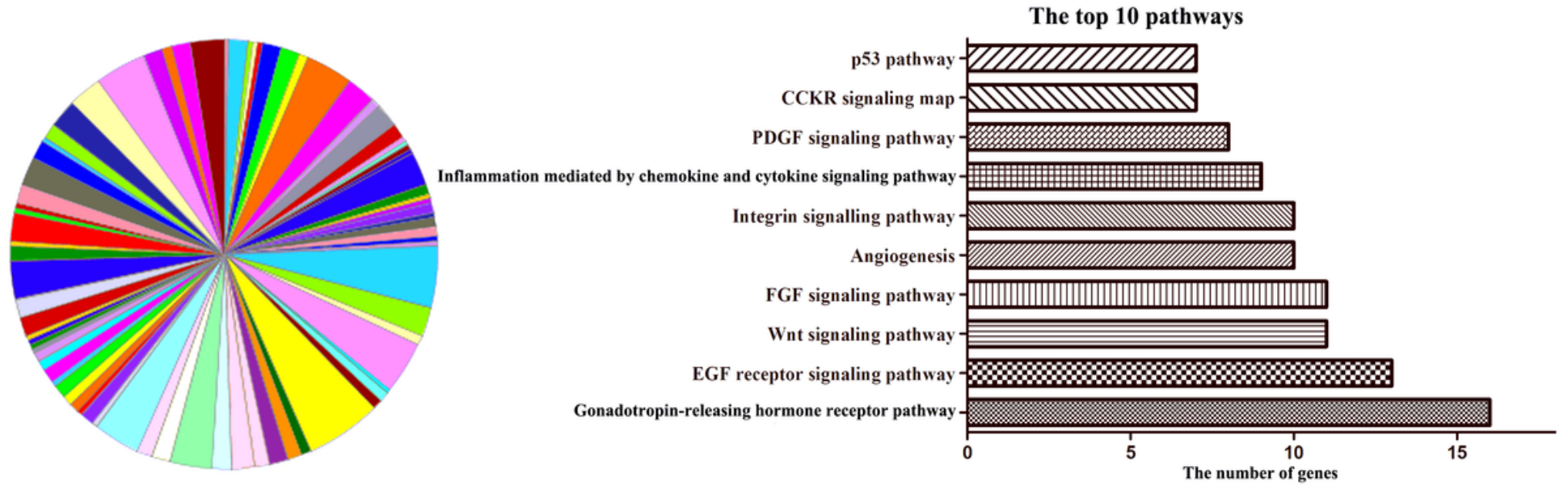

Figure 4

KEGG enrichment analysis was performed on the host genes of common differentially expressed circRNAs in the CV-A16-infected groups versus the control groups. The top 10 significantly enriched pathways are displayed. The vertical lines correspond to the name of pathways, and the horizontal 
line represents the number of genes.

A

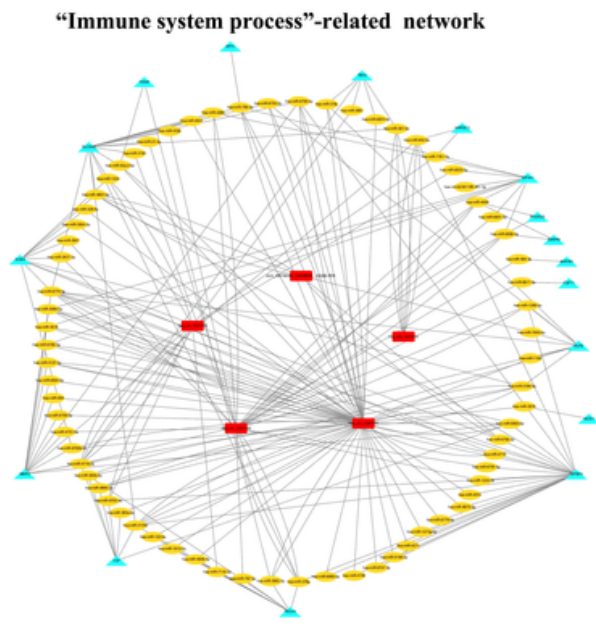

B

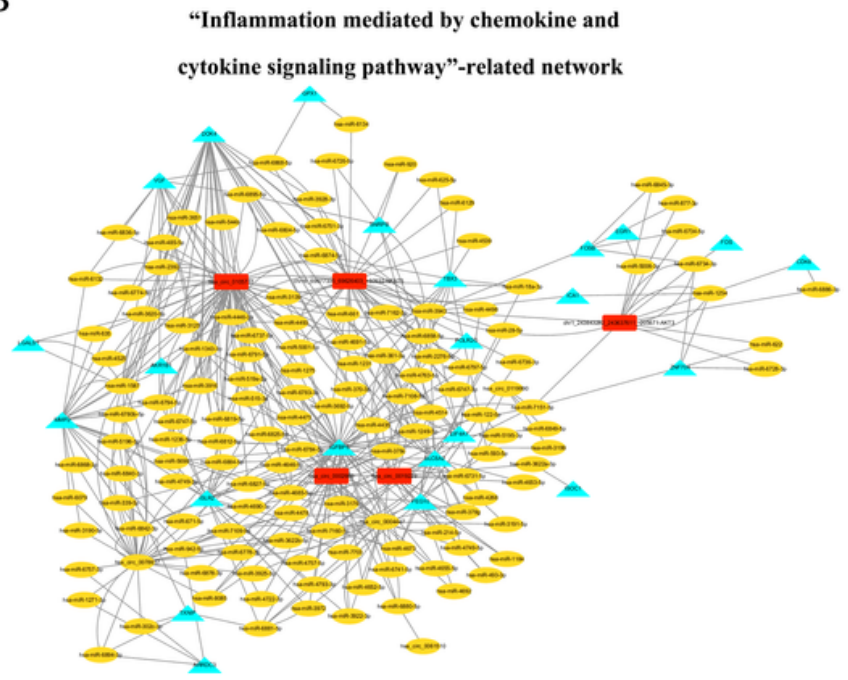

C

"Immune system process"-related target genes
"Inflammation mediated by chemokine and cytokine signaling pathway"-related target genes

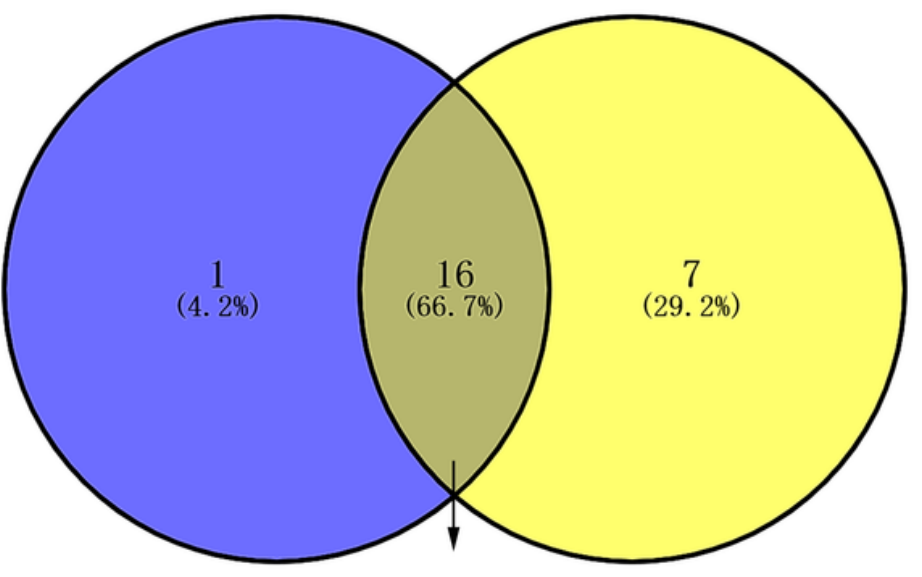

EIFAA1, SLCGA2, TBX3, ARRDC3

IGFBPS. MMP2, PEG10. POLR2G

SNRPB. ISLR2, DOKA. AKRIB1
VGF. TXNIP, GPX1, ISOCI

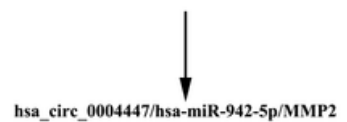

hsa_circ_0078617/hsa-miR-6780b-5p/MMP2 hsa_circ_0078617/hsa-miR-5196-5p/MMP2

\section{Figure 5}

circRNA-miRNA-mRNA interaction network diagram. The network consists of circRNAs (red round rectangles), miRNAs (yellow Ellipse), and their target genes (blue triangles). Based on the prediction and bioinformatics analysis, we showed the circRNA-miRNA-mRNA triple network associated with "Immune system process" in GO-BPs (A) and "Inflammation mediated by chemokine and cytokine signaling pathway" in KEGG analysis (B). Moreover, the overlapping target genes were further screened and the key ceRNA interactions were found (C).

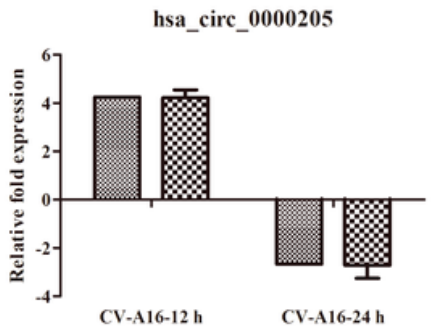

hsa_circ_0002483

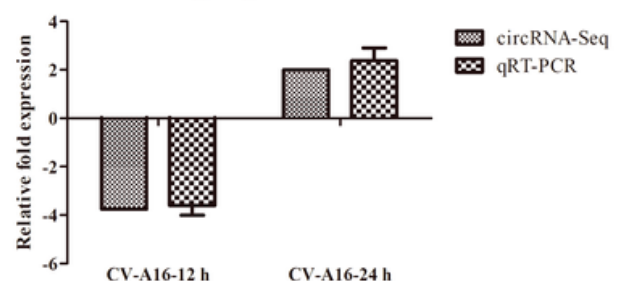

hsa_circ_0002485

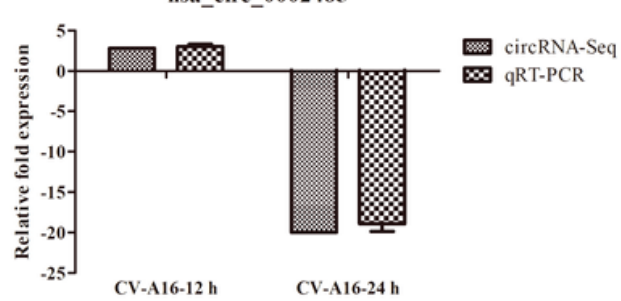

hsa_circ_0002301

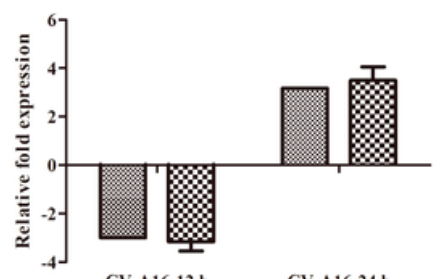

CV-A16-12 h $\quad$ CV-A16-24 h

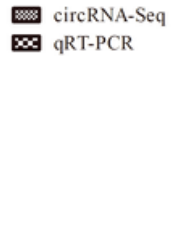

hsa_circ_0007059

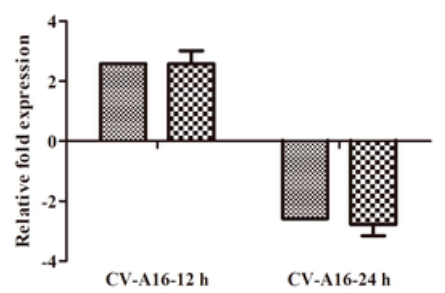

circRNA-Sec $\mathbf{\infty}$ qRT-PCR

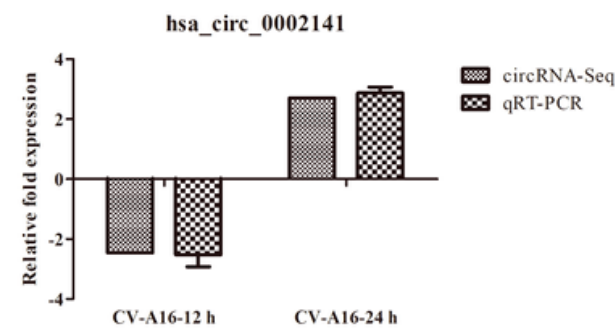




\section{Figure 6}

Validation of selected circRNAs. Six circRNAs were significantly amplified by qRT-PCR and basically consistent with the microarray results. 